\title{
Images in Clinical Medicine: Erythema Migrans
}

Jack Stewart MD, Luis Taboada MD, Joanne Kim MD

\section{Case Report}

Patient is a 30 year old male, with no significant past medical history, who was attending an outdoor party in a wooded area of New Jersey two weeks prior to this hospital admission. Three days following the party, patient noted several papules on his lateral left calf with surrounding erythema. The following day he went to the emergency department and was diagnosed with bacterial cellulitis (Figure 1).

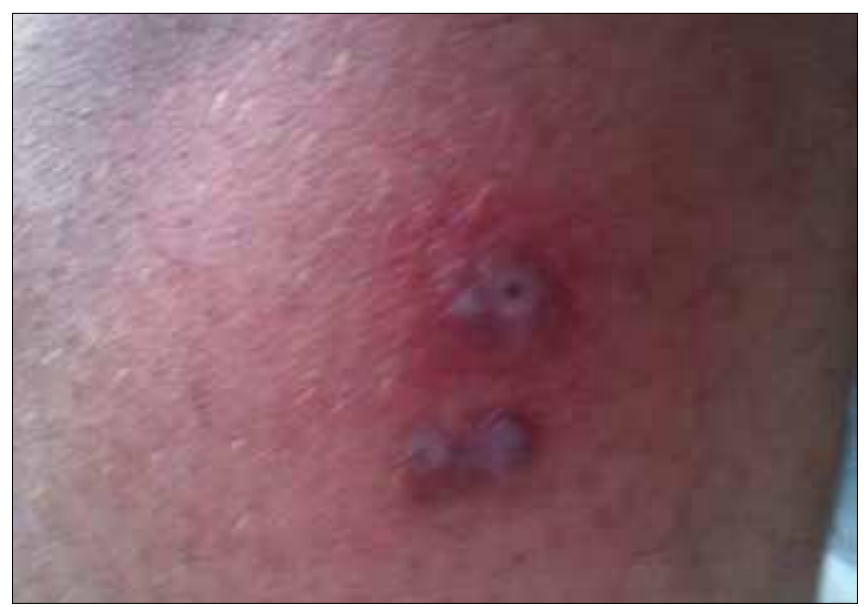

Figure 1. Rash located on lateral left calf on initial presentation.

Patient was discharged home from the emergency department with a 14 day course of Bactrim DS. However two days following his emergency room visit the rash had progressed despite adherence to the antibiotics prescribed. At that time, patient returned to the emergency department (Figure 2).

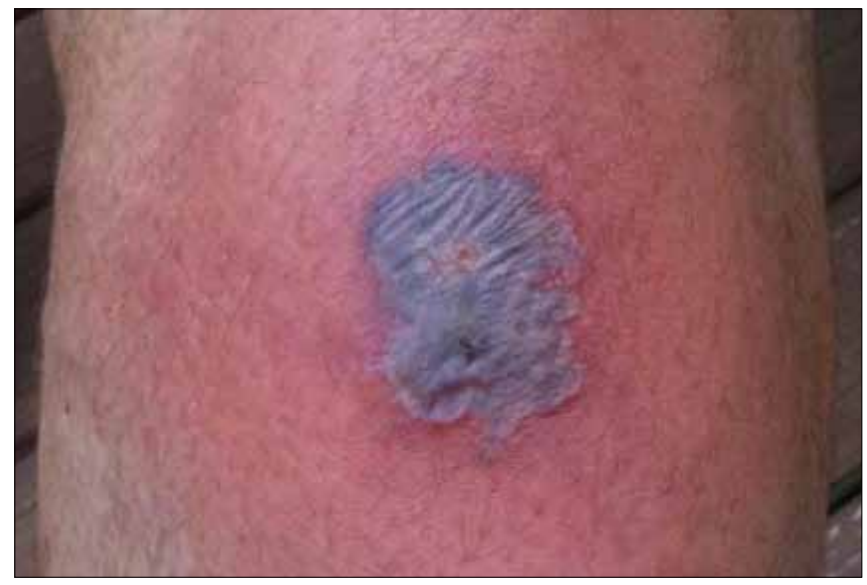

Figure 2. Left lateral calf rash following two days of Bactrim.

The patient was presumed to have either a refractory cellulits secondary to skin flora or infection with Borrelia burgdorferi given his clinical history. Patient was started on broad spectrum antibiotics and doxycycline. The following day, rash was revaluated (Figure 3) and he was diagnosed with erythema migrans.

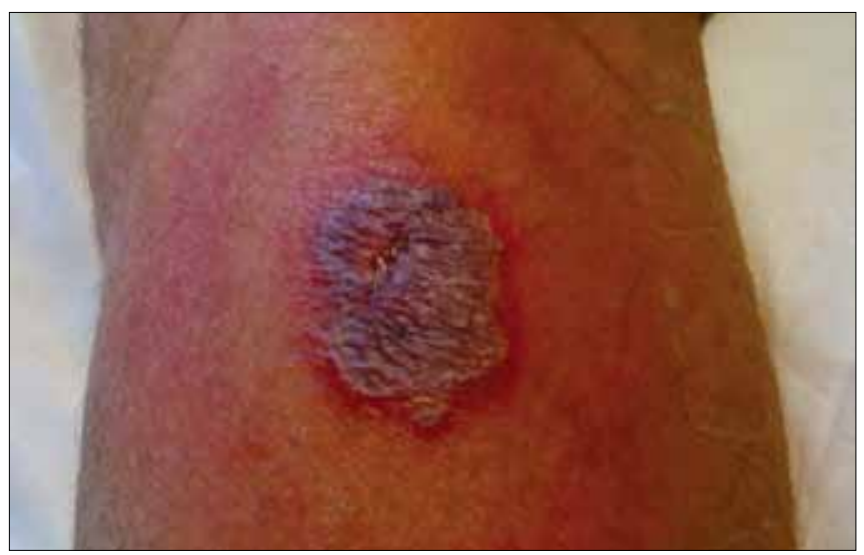

Figure 3. Left lateral calf rash on day 2 of admission.

Patient's condition improved quickly and he was discharged home on a 28 day course of doxycycline.

\section{Discussion}

Erythema migrans occurs in approximately 80 percent of patients and generally manifests within one month following the tick bite. Although the lesions of erythema migrans are typically described as a "bull's eye" and central clearing is considered classic, it often requires considerable expansion of the lesion and, in some instances, is not present early on in the illness. Thus in the first days eythema migrans lesions may be uniformly red.

In light of this information, it is important to consider a wide differential, especially in the early stages of the disease. In addition to Lyme disease, other tick borne illness producing rash such as Rocky Mountain spotted fever, ehrlichiosis, and tularemia should be considered.

Despite the availability of an effective vaccine, measles (rubeola) can present as a blanching erythematous maculopapular rash beginning in the head and neck area and spreading down to the trunk and extremities. The rash of infectious mononucleosis is usually over the trunk but can involve the extremities, including the hands and feet. Acute retroviral syndrome occurring 2-4 weeks after primary HIV infection can manifest as a transient, maculopapular, nonpruritic rash that is usually truncal or facial in location. Approximately 20 percent of cases of erythema infectiosium occur in adults and can manifest with a rash described as first macular and then lacy and reticulated, spreading initially from the limbs to the trunk and buttocks. Additionally, mycoplasma infection may be accompanied by 
skin findings ranging from a mild erythematous maculopapular or vesicular rash to Stevens-Johnson syndrome.

Treatment options for lyme disease differ depending on the clinical scenario. For erythema migrans, amoxicillin is as effective as doxycycline and is preferred for children and pregnant or lactating women. Oral antibiotic treatment for 14-21 days shortens the duration of the rash and generally prevents development of late sequelae. Cefuroxime is also effective, but significantly more expensive. For patients with facial nerve palsy alone, oral doxycycline or amoxicillin may be effective. Patients with other neurologic involvement, such as meningitis, cranial nerve palsies, radiculopathy or cognitive deficits, should be treated with IV ceftriaxone or cefotaxime for 14-28 days.

In patients with minor cardiac conduction disease associated with Lyme disease treatment with oral doxycycline or amoxicillin should be sufficient; however, for those patients with more severe cardiac involvement, intravenous ceftriaxone or cefotaxime is recommended.

Oral therapy with doxycycline or amoxicillin for 28 days is usually effective for treatment of Lyme arthritis. Patients who have not responded to oral treatment may respond to a second course of oral therapy or to intravenous therapy with ceftriaxone or cefotaxime.

\section{References}

1. Wormser, GP et al. Duration of antibiotic therapy for early Lyme disease. A randomized, double-blind, placebo-controlled trial. Ann Intern Med 2003; 138:697.

2. Halperin, JJ. Central nervous system Lyme disease. Curr Infect Dis Rep 2004; 6:298.

3. Dattwyler, RJ et al. A comparison of two treatment regimens of ceftriaxone in late Lyme disease. Wien Klin Wochenschr, 2005; 117:393-7.

“Arc de Triumpe” Photograph by Cecilia Kelly, MD

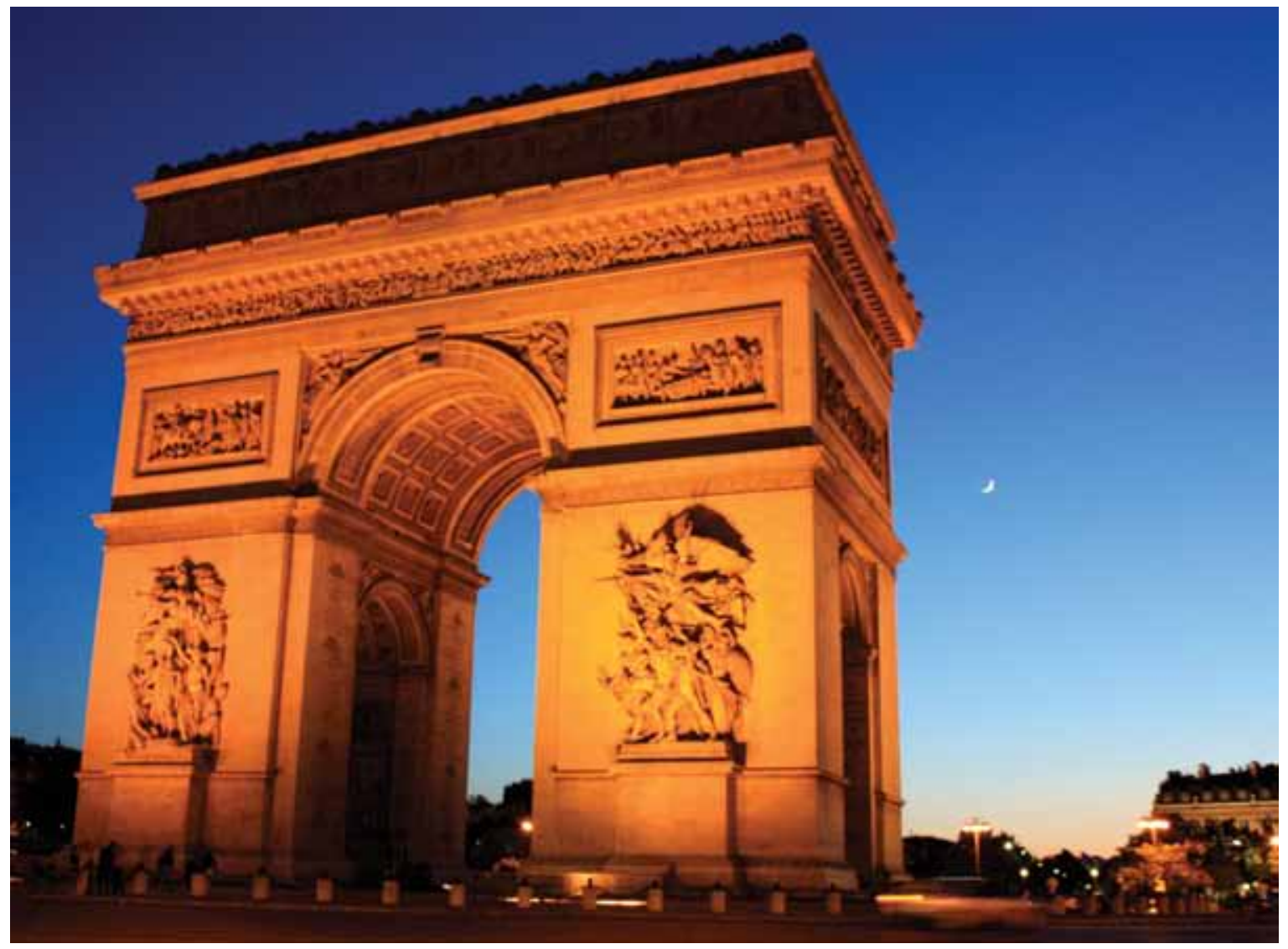

